\title{
Response on Uptake of Nutrients and on Grain Yield from Rice Husk Biochar Application on Oryza sativa L. Grown in a Low Yielding Granary Area of Tanjung Karang, Selangor, Malaysia
}

\author{
DENIEL SANG ${ }^{3}$, ROSENANI ABU BAKAR ${ }^{1}$, SITI HAJAR AHMAD ${ }^{2}$, OSUMANU \\ HARUNA AHMED ${ }^{3}$, WAN ASRINA WAN YAHAYA ${ }^{3}$, AND KHAIRUDDIN ABDUL \\ RAHIM $^{4}$ \\ ${ }^{1}$ Department of Soil Management, Faculty of Agriculture, Universiti Putra Malaysia, 43400 UPM \\ SERDANG, Selangor Darul Ehsan, Malaysia. \\ ${ }^{2}$ Department of Crop Science, Faculty of Agriculture, Universiti Putra Malaysia, 43400 UPM \\ SERDANG, Selangor Darul Ehsan, Malaysia. \\ ${ }^{3}$ Department of Crop Science, Faculty of Agriculture Science and Forestry, Bintulu Sarawak Campus, \\ Universiti Putra Malaysia, 97008 BINTULU, Sarawak, Malaysia. \\ ${ }^{4}$ Division of Agrotechnology and Biosciences, Malaysian Nuclear Agency, Bangi, 43000 KAJANG, \\ Selangor Darul Ehsan, Malaysia.
}

\begin{abstract}
Rice (Oryza sativa L.) production plays a major role in enhancing food security in Malaysia. Lower rice yield and improper soil management practices have raised serious concerns about rice cultivation in Malaysia. The objective of this study was to examine the short-term effects of rice husk biochar (RHB) application on rice yields production on low yielding area for two crop cycles. RHB was applied at the rates of $0,5,10$, and 20 $\mathrm{Mg} \mathrm{ha}^{-1}$. Rice husk biochar was applied one week before rice seeds, of variety MR 263, were directly seeded. Results of the study showed that RHB significantly increased grain yield by $44 \%$ and $46 \%$ in first and second crop cycles, respectively, as compared to the control treatment. Likewise, RHB amended plots showed significant improvement of rice yield components, viz. productive tiller, panicle length, and weight per panicle, than those of the control plots in the first and second crop cycles. Furthermore, RHB significantly increased nitrogen $(\mathrm{N})$, phosphorus $(\mathrm{P})$ and potassium $(\mathrm{K})$ uptake by $17 \%, 18 \%, 29 \%$, respectively, in the first crop cycle, and 26\%,23\%, 110\%, respectively, in the second crop cycle. RHB application also significantly improved soil available $\mathrm{P}$, exchangeable $\mathrm{K}$ and exchangeable $\mathrm{Mg}$ in the two crop cycles. Another interesting finding was that the use of RHB reduced soil total carbon loss by $4 \%$ to $12 \%$ compared with $19 \%$ by the control treatment. These findings suggest that RHB can potentially be used as a soil amendment to increase rice yield production, enhance soil nutrient availability and nutrient uptake, as well as reduce carbon losses especially during drought period.
\end{abstract}

Keywords: rice husk biochar, carbonized biomass; grain yield; nutrient uptake and availability, rice (Oryza sativa L.); soil amendment

\section{INTRODUCTION}

In Malaysia, abundance of rice husk $(408,000 \mathrm{Mg}$ per annum) produced after

Correspondence: Deniel Sang - Department of Crop Science, Faculty of Agriculture Science and Forestry, Sarawak Campus, Universiti Putra Malaysia, 97008, Sarawak, Malaysia. Tel: +6012-878 4987; Email: denielsg@gmail.com

Journal of Wetlands Environmental Management

Vol 9, No 1 (2021) 38 - 52

http://dx.doi.org/10.20527/jwem.v9i1.255 each harvest period, approximately $20 \%$ of crop residue, is burnt as a source of heat energy for drying rice and for rice husk biochar (RHB) production (BERNAS, 2020). The RHB produced has a lower carbon (C) content than pyrolyzed biochar. However, rice husk and RHB are not fully utilized and caused environmental pollution. The application of RHB as soil 
amendment in the rice soils near the mills is deemed feasible, logistically, for the improvement of rice crop and to reduce greenhouse gases (GHGs) emission due to volatilization and denitrification processes.

Rice (Oryza sativa L.) is mainly grown under flooded ecosystem mainly in the coastal areas of the total lowland rice cultivation area in Malaysia, and out of which $83 \%$ is in Peninsular Malaysia, $11 \%$ in Sarawak and $6 \%$ in Sabah, Malaysia (Paddy Statistic of Malaysia, 2015). Total rice production for Malaysia in 2019 was estimated at $1825 \mathrm{Mg}$ within an area of 700000 ha, with national average yield of $4 \mathrm{Mg} \mathrm{ha}^{-1}$ (Index Mundi, 2020). The total domestic consumption is approximately $2775 \mathrm{Mg}$ and Malaysia imported about $1000 \mathrm{Mg}$ in 2019. Department of Statistics Malaysia (2020) reported that as of 2018, Malaysia had achieved $70 \%$ of rice selfsufficiency.

One of the main reasons for low yield of rice in Malaysia is improper soil management practices which lead to high nitrogen $(\mathrm{N})$ loss through volatilization and denitrification processes. Soil amelioration is one of the solutions which has been reported to improve crop performance as well as the yield production. Currently, conversion of crop residues into charcoal, known as biological charcoal (biochar) is gaining attention due to its potential as soil amendment, in addition to abating climate change through sequestering carbon and reducing methane $\left(\mathrm{CH}_{4}\right)$ and nitrous oxide $\left(\mathrm{N}_{2} \mathrm{O}\right)$ emissions (Lehmann et al. 2007; Woolf et al. 2010). Biochar is a byproduct of residual carbonization of biomass after separation from combustible gases under anaerobic condition and high-temperature pyrolysis (Yang et al, 2020). Lehmann and
Joseph (2009) reported that incorporation of biochar helps to conserve and sustain soil productivity as well as reducing environmental impact. As reported in previous studies, application of biochar was found to have dominant effect especially on soil chemical properties rather than crop yield, which was probably due to the application of organic or synthetic fertilizers (Steiner et al., 2007; Asai et al., 2009; Haefele et al., 2011). Thus, biochar application in cultivated land is beneficial in the long-term especially in subtropical and tropical regions due to its roles in improving soil physicochemical properties, organic matter, crop production, and fertilizer use efficiency (Van Zwieten et al., 2010; Deenik et al., 2011). Increase in rice crop yield with biochar application has been reported elsewhere (Steiner et al. 2007; Zhang et al. 2010; de Melo Carvalho et al. 2014).

Rice grain yield from the area of study has been reported to achieve up to $10 \mathrm{Mg} \mathrm{ha}^{-1}$ (Personal communication, 2014) with heavy applications of chemical fertilizers by rich farmers. There were several areas that produced yields of $5 \mathrm{Mg} \mathrm{ha}^{-1}$ and below, including the area where the present study was conducted. However, documented reports on the influence of biochar application on rice yield is still greatly lacking, especially in Malaysia. We hypothesized that RHB application helps to increase rice yield through improvement of soil properties and nutrient uptake in areas with low rice productivity. The objective of this study was to examine the short-term effects of RHB application on rice yields in low yielding areas for two crop cycles. 
MATERIALS AND METHODS

\section{Field sites}

A field experiment was established in a Barat Laut Selangor rice area in Kampung Tengah Pasir Panjang $\quad\left(3^{\circ} 35^{\prime} 27.6 " \mathrm{~N}\right.$ $\left.101^{\circ} 04^{\prime} 10.5^{\prime \prime} \mathrm{E}\right)$, over two rice cropping seasons. Total rainfall was $517 \mathrm{~mm}$ in the first crop cycle (September to December 2014) and $321 \mathrm{~mm}$ in the second crop cycle (March to June 2015), whereas the average temperature during this period of study was $27^{\circ} \mathrm{C}$. A long drought during the second crop cycle affected the water level during the rice growth period. Rice cultivation in this area has been carried out for the past 39 years. Soil of the experimental field is of Sabrang series, which is classified as Sulfic Endoaquepts. The soil has a clay texture with $\mathrm{pH}$ of 5.50, and the topsoil has total carbon content of $172 \mathrm{~g} \mathrm{~kg}^{-1}$ with total mineral content of $80 \%$ ( $1.65 \%$ sand, $38.83 \%$ silt, and $60.47 \%$ clay) as shown in Table 1.

Table 1.Selected chemical properties of the soil before treatment and rice husk biochar (RHB) properties

\begin{tabular}{lccc}
\hline Chemical properties & $\begin{array}{c}\text { Initial soil } \\
\text { (non-amended soil) }\end{array}$ & $\begin{array}{c}\text { RHB } \\
\text { (First } \\
\text { crop cycle) }\end{array}$ & $\begin{array}{c}\text { RHB } \\
\text { (Second } \\
\text { crop cycle) }\end{array}$ \\
\hline $\mathrm{pH}($ Flood water) & 6.30 & $-*$ & - \\
$\mathrm{pH}($ soil = $1: 2.5 ;$ biochar $=1: 50)$ & 5.50 & 8.50 & 7.4 \\
Total Carbon $\left(\mathrm{g} \mathrm{kg}^{-1}\right)$ & 172.00 & 210.00 & 431.20 \\
Total Nitrogen $\left(\mathrm{g} \mathrm{kg}^{-1}\right)$ & 6.00 & 1.00 & 6.80 \\
Available P $\left(\mathrm{mg} \mathrm{kg}^{-1}\right)$ & 64.60 & - & - \\
Exchangeable K $\left(\mathrm{mg} \mathrm{kg}^{-1}\right)$ & 471.9 & - & - \\
Exchangeable Ca $\left(\mathrm{mg} \mathrm{kg}^{-1}\right)$ & 2646 & - & - \\
Exchangeable Mg $\left(\mathrm{mg} \mathrm{kg}^{-1}\right)$ & 559.2 & - & - \\
CEC $\left(\mathrm{cmol}_{(+)} \mathrm{kg}^{-1}\right)$ & 15.90 & - & 60 \\
Total ash $(\%)$ & - & 88.50 & 16 \\
Volatile matter $(\%)$ & - & 9.20 & 1.55 \\
Total P $\left(\mathrm{g} \mathrm{kg}^{-1}\right)$ & - & 5.10 & 2.92 \\
Total K $\left(\mathrm{g} \mathrm{kg}^{-1}\right)$ & - & 2.70 & 0.85 \\
Total Ca $\left(\mathrm{g} \mathrm{kg}^{-1}\right)$ & - & 0.98 & 0.87 \\
Total Mg $\left(\mathrm{g} \mathrm{kg}^{-1}\right)$ & - & 0.20 & \\
\hline
\end{tabular}

*: not determined 
The RHB used for the field experiment was produced by a carbonization process which was collected from SENDI Enterprise at Sekinchan, Selangor. RHB treatment was applied at the rates of $0,5,10$ and $20 \mathrm{Mg} \mathrm{ha}^{-1}$ at the beginning of each crop cycle. RHB was spread onto the surface during land preparation after second ploughing and then mixed into soil by ploughing to a depth of $15 \mathrm{~cm}$. The four treatments were arranged in a fully randomized complete block design (RCBD) with four replications and plot size of $4 \mathrm{~m} \times 4 \mathrm{~m}$. Rice variety MR 263 was used and sown through direct broadcasting $\left(200 \mathrm{~g} \mathrm{plot}^{-1}\right)$. The plot was irrigated 14 days after broadcast of seeds and then drained at the ripening stage at 100 days after sowing (DAS). Fertilizer application of the rice crop was according to the recommended rates and timing by Sistem Penanaman Padi Lestari (MARDI, 2008.

\section{Plant and Soil Sampling and Analysis}

Rice was harvested at 110 days after sowing. Sampling with $1 \mathrm{~m}^{2}$ quadrant was carried out to determine rice yield components such grain yield (GY), number of productive tillers (PT), number of panicles (NP), percent filled grains (FG), weight of 1000 grains, panicle length (PL), weight per panicle (WPP), straw dry matter weight (DMWs) and harvest index (HI). Three points of composite soil samples were taken before applying treatment for soil characterization and after harvest to determine the soil $\mathrm{pH}$, total carbon (TC), total nitrogen (TN), available phosphorus (P), exchangeable potassium $(\mathrm{K})$, calcium $(\mathrm{Ca})$, magnesium $(\mathrm{Mg})$ and soil cation exchange capacity (CEC). Harvested plant sample was oven-dried at $65{ }^{\circ} \mathrm{C}$ to constant weight before weighing to determine the dry matter weight and soils were air-dried, ground and sieved with 2 $\mathrm{mm}$ mesh. The oven-dried plant samples were ground into a $0.25 \mathrm{~mm}$ size and subjected to wet digestion before being analyzed for macronutrients $(\mathrm{P}, \mathrm{K}, \mathrm{Ca}$, and $\mathrm{Mg})$. Soil $\mathrm{pH}$ was determined using a $\mathrm{pH}$ meter with 1:2.5 ratio soil solutions with deionized water. Total $\mathrm{C}$ (soil) and total $\mathrm{N}$ (soil and plant samples) were determined using LECO TruMac CNS analyzer. The soil available $\mathrm{P}$ was determined using the method of Bray and Kurtz (1945). Exchangeable cations ( $\mathrm{K}, \mathrm{Ca}$ and $\mathrm{Mg}$ ) and soil $\mathrm{CEC}$ were determined using ammonium acetate of $\mathrm{pH}$ 7.0. For RHB analysis, LECO TruMac CNS analyzer was used to measure the RHB total $\mathrm{C}$ and total $\mathrm{N}$. The $\mathrm{pH}$ of $\mathrm{RHB}$ was determined in water at ratio of 1:5 (w/v). Total elements $(\mathrm{P}, \mathrm{K}, \mathrm{Ca}$, and $\mathrm{Mg}$ ) were determined using the dry ashing method. Volatile matter and ash content were measured using ASTM D 3172 Standard practice for Proximate Analysis of Coal and Coke.

\section{Statistical Analysis}

One-way analysis of variance (ANOVA) was performed to determine the significant differences in yield parameters, nutrient uptake and soil properties between different treatments. The differences in means were separated by least significant difference (LSD) test at $p \leq 0.05$. Pearson correlation coefficients were calculated to determine the relationship between GY and nutrient uptake, soil properties and yield parameters using SAS 9.2 software (SAS Institute Inc., USA). 
ISSN: 2477-5223 (Online)
Data describing the chemical properties at the end of field experiment under different rates of RHB treatments are presented in Table 2.

Table 2. Soil chemical characteristic as influenced by RHB application in first and second crop cycle in highly organic topsoil (0-15 cm depth)

\begin{tabular}{|c|c|c|c|c|c|c|c|c|}
\hline \multirow{2}{*}{ Variables } & \multicolumn{4}{|c|}{ First crop cycle (Mg ha ${ }^{-1}$ RHB) } & \multicolumn{4}{|c|}{ Second crop cycle $\left(\mathrm{Mg} \mathrm{ha}^{-1}\right.$ RHB) } \\
\hline & $\mathbf{0}$ & 5 & 10 & 20 & $\mathbf{0}$ & 5 & 10 & 20 \\
\hline $\mathrm{pH}$ & $\begin{array}{l}5.24 \mathrm{c}^{*} \\
( \pm 0.07)\end{array}$ & $\begin{array}{c}5.77 \mathbf{a} \\
( \pm 0.07)\end{array}$ & $\begin{array}{c}5.75 \mathbf{a} \\
( \pm 0.03)\end{array}$ & $\begin{array}{c}5.46 \mathbf{b} \\
( \pm 0.08)\end{array}$ & $\begin{array}{c}5.62 \mathbf{a} \\
( \pm 0.07)\end{array}$ & $\begin{array}{c}5.59 \mathbf{a} \\
( \pm 0.10)\end{array}$ & $\begin{array}{c}5.57 \mathbf{a} \\
( \pm 0.04)\end{array}$ & $\begin{array}{c}5.51 \mathbf{a} \\
( \pm 0.09)\end{array}$ \\
\hline Total C $\left(\mathrm{g} \mathrm{kg}^{-1}\right)$ & $\begin{array}{l}166.7 \mathbf{a} \\
( \pm 3.75)\end{array}$ & $\begin{array}{l}167.4 \mathbf{a} \\
( \pm 5.62)\end{array}$ & $\begin{array}{l}166.0 \mathbf{a} \\
( \pm 3.98)\end{array}$ & $\begin{array}{l}164.6 \mathbf{a} \\
( \pm 2.63)\end{array}$ & $\begin{array}{l}139.7 \mathbf{c} \\
( \pm 2.66)\end{array}$ & $\begin{array}{c}155.9 \mathbf{a} \\
( \pm 1.30)\end{array}$ & $\begin{array}{c}149.7 \mathbf{b} \\
( \pm 0.71)\end{array}$ & $\begin{array}{c}153.3 \mathbf{a} \\
( \pm 1.53)\end{array}$ \\
\hline Total N $\left(\mathrm{g} \mathrm{kg}^{-1}\right)$ & $\begin{array}{c}6.6 \mathbf{a} \\
( \pm 0.21)\end{array}$ & $\begin{array}{c}6.7 \mathbf{a} \\
( \pm 0.10)\end{array}$ & $\begin{array}{c}6.5 \mathbf{a} \\
( \pm 0.30)\end{array}$ & $\begin{array}{c}6.7 \mathbf{a} \\
( \pm 0.20)\end{array}$ & $\begin{array}{c}5.1 \mathbf{c} \\
( \pm 0.14)\end{array}$ & $\begin{array}{c}5.5 \mathbf{b} \\
( \pm 0.05)\end{array}$ & $\begin{array}{c}4.7 \mathbf{d} \\
( \pm 0.11)\end{array}$ & $\begin{array}{c}6.2 \mathbf{a} \\
( \pm 0.01)\end{array}$ \\
\hline Available $\mathrm{P}\left(\mathrm{mg} \mathrm{kg}^{-1}\right)$ & $\begin{array}{l}72.75 \mathbf{b} \\
( \pm 2.52)\end{array}$ & $\begin{array}{l}72.82 \mathbf{b} \\
( \pm 3.95)\end{array}$ & $\begin{array}{l}76.30 \mathbf{b} \\
( \pm 1.71)\end{array}$ & $\begin{array}{l}91.07 \mathbf{a} \\
( \pm 3.14)\end{array}$ & $\begin{array}{c}55.13 \mathbf{b} \\
( \pm 0.79)\end{array}$ & $\begin{array}{l}60.02 \mathbf{a} \\
( \pm 2.90)\end{array}$ & $\begin{array}{l}61.64 \mathbf{a} \\
( \pm 2.63)\end{array}$ & $\begin{array}{l}62.04 \mathbf{a} \\
( \pm 3.23)\end{array}$ \\
\hline Exch. K (mg kg $\left.{ }^{-1}\right)$ & $\begin{array}{c}150 \mathbf{c} \\
( \pm 19.57)\end{array}$ & $\begin{array}{c}174 \mathbf{c} \\
( \pm 7.69)\end{array}$ & $\begin{array}{c}222 \mathbf{b} \\
( \pm 36.87)\end{array}$ & $\begin{array}{c}256 \mathbf{a} \\
( \pm 7.84)\end{array}$ & $\begin{array}{c}3740 \mathbf{d} \\
( \pm 56.34)\end{array}$ & $\begin{array}{c}4772 \mathbf{b} \\
( \pm 50.67)\end{array}$ & $\begin{array}{c}5192 \mathbf{a} \\
( \pm 92.73)\end{array}$ & $\begin{array}{c}3906 \mathbf{c} \\
( \pm 28.84)\end{array}$ \\
\hline Exch. $\mathrm{Mg}\left(\mathrm{mg} \mathrm{kg}^{-1}\right)$ & $\begin{array}{c}342 \mathbf{c} \\
( \pm 1.35)\end{array}$ & $\begin{array}{c}373 \mathbf{b} \\
( \pm 3.86)\end{array}$ & $\begin{array}{c}405 \mathbf{a} \\
( \pm 12.28)\end{array}$ & $\begin{array}{c}353 \mathbf{c} \\
( \pm 8.14)\end{array}$ & $\begin{array}{c}742 \mathbf{b} \\
( \pm 12.86)\end{array}$ & $\begin{array}{c}821 \mathbf{a} \\
( \pm 25.23)\end{array}$ & $\begin{array}{c}829 \mathbf{a} \\
( \pm 29.37)\end{array}$ & $\begin{array}{c}829 \mathbf{a} \\
( \pm 29.37)\end{array}$ \\
\hline Exch. Ca $\left(\mathrm{mg} \mathrm{kg}^{-1}\right)$ & $\begin{array}{c}3514 \mathbf{a} \\
( \pm 126.92)\end{array}$ & $\begin{array}{c}3605 \mathbf{a} \\
( \pm 108.18)\end{array}$ & $\begin{array}{c}3646 \mathbf{a} \\
( \pm 139.27)\end{array}$ & $\begin{array}{c}3655 \mathbf{a} \\
( \pm 114.02)\end{array}$ & $\begin{array}{c}108 \mathbf{c} \\
( \pm 4.80)\end{array}$ & $\begin{array}{c}109 \mathbf{c} \\
( \pm 11.96)\end{array}$ & $\begin{array}{c}154 \mathbf{a} \\
( \pm 12.26)\end{array}$ & $\begin{array}{c}135 \mathbf{b} \\
( \pm 10.16)\end{array}$ \\
\hline $\operatorname{CEC}\left(\operatorname{cmol}\left({ }_{+}\right) \mathrm{kg}^{-1}\right)$ & $\begin{array}{l}67.52 \mathbf{a} \\
( \pm 4.91)\end{array}$ & $\begin{array}{l}73.04 \mathbf{a} \\
( \pm 3.35)\end{array}$ & $\begin{array}{l}73.77 \mathbf{a} \\
( \pm 2.90)\end{array}$ & $\begin{array}{l}74.73 \mathbf{a} \\
( \pm 6.15)\end{array}$ & $\begin{array}{l}30.08 \mathbf{a} \\
( \pm 2.84)\end{array}$ & $\begin{array}{l}30.61 \mathbf{a} \\
( \pm 3.00)\end{array}$ & $\begin{array}{l}30.77 \mathbf{a} \\
( \pm 2.95)\end{array}$ & $\begin{array}{l}32.09 \mathbf{a} \\
( \pm 2.42)\end{array}$ \\
\hline
\end{tabular}

* Means with different letters indicates significantly difference between treatment at 0.05 level by

LSD test and \pm value indicate mean standard deviation.

RHB amendment significantly increased soil $\mathrm{pH}$ in the first crop cycle, whereas no significant effect was detected in the second crop cycle. It was observed that soil $\mathrm{pH}$ in the first crop cycle increased up to $10 \%$ with $5 \mathrm{Mg}$ $\mathrm{ha}^{-1}$ RHB application compared to control. However, there was no significant effect of RHB on soil TC in the first and second crop cycle. However, there was significant difference of $\mathrm{TC}$ in the second crop cycle in comparison to to unamended plots. A small increase of soil TC (7-11\%) as compared to unamended plots was observed during the second crop cycle. As observed for soil total $\mathrm{N}$, there was no significant response in the first crop cycle, but found significantly influenced soil total $\mathrm{N}$ in the second crop cycle. Soil total $\mathrm{N}$ increased under RHB treated soil with peak at $20 \mathrm{Mg} \mathrm{ha}^{-1}$ (6.28 $\mathrm{g} \mathrm{kg}^{-1}$ ) in the second crop cycle. Application of RHB was statistically significant only for the $20 \mathrm{Mg} \mathrm{ha}^{-1}$ in the first crop cycle, while significantly increased under all RHB treatment in the second crop cycle. In the first crop cycle, soil exchangeable $\mathrm{K}$ was significantly affected by RHB at rate of 10 and $20 \mathrm{Mg} \mathrm{ha}^{-1}$, while significantly increased in all RHB treatments compared to control treatment. A

Journal of Wetlands Environmental Management

Vol 9, No 1 (2021) 38 - 52 
similar result was observed for exchangeable $\mathrm{Mg}$ in the second crop cycle, whereas a significant difference only found at application of 5 and 10 $\mathrm{Mg} \mathrm{ha}^{-1}$ during the first crop cycle. However, RHB amendment only significantly influenced soil exchangeable $\mathrm{Ca}$ during the second crop cycle with application rates of 10 and $20 \mathrm{Mg}$ $\mathrm{ha}^{-1}$. Furthermore, soil CEC was not significantly different between plots with or without RHB applications for both crop cycles.

\section{Effects of RHB Amendment on Nutrient Uptake}

Rice husk biochar addition significantly $(P \leq 0.05)$ increased aboveground biomass $\mathrm{N}$ uptake as compared to the control in both crop cycles (Table 3 ).

Table 3. Total nutrient uptake by aboveground biomass after rice husk biochar (RHB) application at different rates in first and second crop cycles

\begin{tabular}{|c|c|c|c|c|c|c|c|c|}
\hline \multirow{2}{*}{ Variables } & \multicolumn{4}{|c|}{ First crop cycle $\left(\mathrm{Mg} \mathrm{ha}^{-1} \mathrm{RHB}\right)$} & \multicolumn{4}{|c|}{ Second crop cycle ( $\left.\mathrm{Mg} \mathrm{ha}^{-1} \mathrm{RHB}\right)$} \\
\hline & 0 & 5 & 10 & 20 & 0 & 5 & 10 & 20 \\
\hline $\mathrm{N}\left(\mathrm{kg} \mathrm{ha}^{-1}\right)$ & $\begin{array}{l}142.60 c^{*} \\
( \pm 2.56)\end{array}$ & $\begin{array}{l}166.70 \mathrm{a} \\
( \pm 3.23)\end{array}$ & $\begin{array}{l}146.91 b \\
( \pm 1.67)\end{array}$ & $\begin{array}{l}145.35 b c \\
( \pm 3.19)\end{array}$ & $\begin{array}{l}119.11 d \\
( \pm 1.62)\end{array}$ & $\begin{array}{l}145.34 b \\
( \pm 3.69)\end{array}$ & $\begin{array}{l}134.93 \mathrm{c} \\
( \pm 3.00)\end{array}$ & $\begin{array}{l}150.23 \mathrm{a} \\
( \pm 2.74)\end{array}$ \\
\hline NHI (\%) & $\begin{array}{l}47.71 \mathrm{c} \\
( \pm 2.78)\end{array}$ & $\begin{array}{l}53.58 \mathrm{~b} \\
( \pm 2.57)\end{array}$ & $\begin{array}{l}57.86 \mathrm{ab} \\
( \pm 3.73)\end{array}$ & $\begin{array}{l}58.89 \mathrm{a} \\
( \pm 1.18)\end{array}$ & - & - & - & - \\
\hline $\mathrm{P}\left(\mathrm{kg} \mathrm{ha}^{-1}\right)$ & $\begin{array}{l}50.79 b \\
( \pm 3.31)\end{array}$ & $\begin{array}{l}59.32 \mathrm{a} \\
( \pm 2.55)\end{array}$ & $\begin{array}{l}60.07 \mathrm{a} \\
( \pm 4.06)\end{array}$ & $\begin{array}{l}49.09 b \\
( \pm 1.39)\end{array}$ & $\begin{array}{l}22.58 \mathrm{c} \\
( \pm 1.25)\end{array}$ & $\begin{array}{l}25.82 b c \\
( \pm 2.75)\end{array}$ & $\begin{array}{l}28.18 \mathrm{ab} \\
( \pm 0.36)\end{array}$ & $\begin{array}{l}29.62 \mathrm{a} \\
( \pm 1.99)\end{array}$ \\
\hline $\mathrm{K},\left(\mathrm{kg} \mathrm{ha}^{-1}\right)$ & $\begin{array}{l}125.83 b \\
( \pm 6.51)\end{array}$ & $\begin{array}{c}161.57 \mathrm{a} \\
( \pm 3.77)\end{array}$ & $\begin{array}{l}162.33 \mathrm{a} \\
( \pm 10.25)\end{array}$ & $\begin{array}{l}156.08 \mathrm{a} \\
( \pm 5.27)\end{array}$ & $\begin{array}{l}116.99 \mathrm{~d} \\
( \pm 2.89)\end{array}$ & $\begin{array}{l}166.37 \mathrm{c} \\
( \pm 3.55)\end{array}$ & $\begin{array}{l}207.57 b \\
( \pm 4.31)\end{array}$ & $\begin{array}{l}245.52 \mathrm{a} \\
( \pm 4.55)\end{array}$ \\
\hline $\mathrm{Ca}\left(\mathrm{kg} \mathrm{ha}^{-1}\right)$ & $\begin{array}{l}10.15 a \\
( \pm 0.95)\end{array}$ & $\begin{array}{l}11.61 \mathrm{a} \\
( \pm 1.47)\end{array}$ & $\begin{array}{l}12.90 \mathrm{a} \\
( \pm 1.83)\end{array}$ & $\begin{array}{l}12.09 \mathrm{a} \\
( \pm 0.54)\end{array}$ & $\begin{array}{l}14.34 \mathrm{a} \\
( \pm 2.86)\end{array}$ & $\begin{array}{l}14.68 \mathrm{a} \\
( \pm 2.73)\end{array}$ & $\begin{array}{l}16.19 a \\
( \pm 1.42)\end{array}$ & $\begin{array}{l}16.94 \mathrm{a} \\
( \pm 1.36)\end{array}$ \\
\hline $\operatorname{Mg}\left(\mathrm{kg} \mathrm{ha}^{-1}\right)$ & $\begin{array}{l}17.46 \mathrm{ab} \\
( \pm 1.46)\end{array}$ & $\begin{array}{l}18.54 \mathrm{a} \\
( \pm 0.85)\end{array}$ & $\begin{array}{l}17.21 \mathrm{~b} \\
( \pm 0.23)\end{array}$ & $\begin{array}{l}15.58 \mathrm{c} \\
( \pm 0.47)\end{array}$ & $\begin{array}{l}12.79 b \\
( \pm 1.09)\end{array}$ & $\begin{array}{l}16.68 \mathrm{a} \\
( \pm 0.22)\end{array}$ & $\begin{array}{l}16.14 a \\
( \pm 0.98)\end{array}$ & $\begin{array}{l}16.37 \mathrm{a} \\
( \pm 0.92)\end{array}$ \\
\hline $\mathrm{Zn}\left(\mathrm{kg} \mathrm{ha}^{-1}\right)$ & $\begin{array}{l}9.08 \mathrm{a} \\
( \pm 1.14)\end{array}$ & $\begin{array}{l}10.01 \mathrm{a} \\
( \pm 0.10)\end{array}$ & $\begin{array}{l}8.54 a \\
( \pm 0.36)\end{array}$ & $\begin{array}{l}9.15 \mathrm{a} \\
( \pm 0.73)\end{array}$ & $\begin{array}{l}10.86 b c \\
( \pm 1.35)\end{array}$ & $\begin{array}{l}14.85 \mathrm{~b} \\
( \pm 0.85)\end{array}$ & $\begin{array}{l}14.89 \mathrm{~b} \\
( \pm 1.26)\end{array}$ & $\begin{array}{l}19.16 \mathrm{a} \\
( \pm 0.80)\end{array}$ \\
\hline
\end{tabular}

* Means with different letters indicates significantly difference between treatment at 0.05 level by LSD test and \pm value indicate mean standard deviation; $\mathrm{N}=$ nitrogen; $\mathrm{NHI}=$ nitrogen harvest index, $\mathrm{P}=$ phosphorus; $\mathrm{K}=$ potassium; $\mathrm{Ca}=\mathrm{Calcium} ; \mathrm{Mg}=$ Magnesium; $\mathrm{Zn}=$ zinc.

RHB treatment indicated that $\mathrm{N}$ uptake by aboveground biomass significantly increased up to $17 \%$ and $26 \%$ in the first and second crop cycle, respectively. It should be noted that Nitrogen Harvest Index (NHI) was only measured in the first crop cycle. With the addition of RHB (20 Mg ha $\left.{ }^{-1}\right)$, significant increases in NHI was observed as compared to control. In the first crop cycle, $\mathrm{P}$ uptake was significantly influenced by application of 5 and $10 \mathrm{Mg} \mathrm{ha}{ }^{-1} \mathrm{RHB}$ as compared to control treatment. The highest value was recorded at application of $10 \mathrm{Mg} \mathrm{ha}^{-1} \mathrm{RHB}\left(60.07 \mathrm{~kg} \mathrm{ha}^{-1}\right)$, whereas the lowest value was recorded at control

Journal of Wetlands Environmental Management

Vol 9, No 1 (2021) 38 - 52

http://dx.doi.org/10.20527/jwem.v9i1.255 
treatment $\left(50.79 \mathrm{~kg} \mathrm{ha}^{-1}\right)$. However, in the second crop cycle RHB treatment was statistically significant with application of 10 and $20 \mathrm{Mg} \mathrm{ha}^{-1}$ compared with the control. The highest reading was recorded at $20 \mathrm{Mg} \mathrm{ha}^{-1}$ $\left(29.62 \mathrm{~kg} \mathrm{ha}^{-1}\right)$. The $\mathrm{K}$ uptake also showed significant differences in all RHB treated plots compared with control in both first and second crop cycle. In contrast, the application of RHB had no effect on $\mathrm{Ca}$ uptake. Similar results were observed for $\mathrm{Mg}$ and $\mathrm{Zn}$ uptake in the first crop cycle. However, there was a significant difference in $\mathrm{Mg}$ and $\mathrm{Zn}$ uptake for the second crop cycle with an increment of $28 \%$ and $76 \%$, respectively.

\section{Effects of RHB Amendment on Yield Parameters}

The effect of RHB application on rice yield parameters shown in Table 4.

Table 4. Effect of rice husk biochar (RHB) on rice yield parameters at first and second crop cycles

\begin{tabular}{lcccccccc}
\hline & \multicolumn{3}{c}{ First crop cycle $\left(\mathrm{Mg} \mathrm{ha}^{-1} \mathrm{RHB}\right)$} & \multicolumn{3}{c}{ Second crop cycle $\left(\mathrm{Mg} \mathrm{ha}^{-1} \mathrm{RHB}\right)$} \\
\cline { 2 - 8 } Variables & & & & & & & & \\
& & 5 & 10 & 20 & 0 & 5 & 10 & 20 \\
& $5.47 \mathrm{~b}^{*}$ & $7.48 \mathrm{a}$ & $7.21 \mathrm{a}$ & $7.89 \mathrm{a}$ & $3.23 \mathrm{~d}$ & $3.53 \mathrm{c}$ & $4.70 \mathrm{a}$ & $4.16 \mathrm{~b}$ \\
GY $\left(\mathrm{Mg} \mathrm{ha}^{-1}\right)$ & $( \pm 0.44)$ & $( \pm 0.13)$ & $( \pm 0.36)$ & $( \pm 0.59)$ & $( \pm 0.17)$ & $( \pm 0.11)$ & $( \pm 0.23)$ & $( \pm 0.18)$ \\
& $80.95 \mathrm{c}$ & $83.38 \mathrm{~b}$ & $86.25 \mathrm{a}$ & $86.00 \mathrm{a}$ & $84.70 \mathrm{~b}$ & $90.59 \mathrm{~b}$ & $90.76 \mathrm{a}$ & $91.45 \mathrm{a}$ \\
PT $(\%)$ & $( \pm 1.88)$ & $( \pm 1.28)$ & $( \pm 0.20)$ & $( \pm 1.97)$ & $( \pm 1.64)$ & $( \pm 1.30)$ & $( \pm 1.87)$ & $( \pm 2.25)$ \\
& $416 \mathrm{~b}$ & $536 \mathrm{a}$ & $413 \mathrm{~b}$ & $411 \mathrm{~b}$ & $306 \mathrm{~d}$ & $332 \mathrm{c}$ & $371 \mathrm{~b}$ & $413 \mathrm{a}$ \\
NP $\left(\mathrm{m}^{-2}\right)$ & $( \pm 10.81)$ & $( \pm 5.12)$ & $( \pm 8.85)$ & $( \pm 10.25)$ & $( \pm 4.66)$ & $( \pm 6.18)$ & $( \pm 7.45)$ & $( \pm 4.11)$ \\
& $82.22 \mathrm{a}$ & $78.46 \mathrm{a}$ & $76.41 \mathrm{a}$ & $78.86 \mathrm{a}$ & $66.63 \mathrm{c}$ & $88.20 \mathrm{a}$ & $77.86 \mathrm{~b}$ & $78.04 \mathrm{~b}$ \\
FG $(\%)$ & $( \pm 3.06)$ & $( \pm 3.20)$ & $( \pm 2.80)$ & $( \pm 0.56)$ & $( \pm 2.25)$ & $( \pm 0.19)$ & $( \pm 3.01)$ & $( \pm 2.95)$ \\
& $10.08 \mathrm{~b}$ & $10.05 \mathrm{~b}$ & $9.80 \mathrm{c}$ & $10.83 \mathrm{a}$ & $11.79 \mathrm{~b}$ & $12.60 \mathrm{a}$ & $13.09 \mathrm{a}$ & $11.92 \mathrm{~b}$ \\
TG $(\mathrm{g})$ & $( \pm 0.13)$ & $( \pm 0.04)$ & $( \pm 0.17)$ & $( \pm 0.18)$ & $( \pm 0.48)$ & $( \pm 0.50)$ & $( \pm 0.42)$ & $( \pm 0.41)$ \\
& $20.32 \mathrm{c}$ & $21.78 \mathrm{ab}$ & $21.05 \mathrm{bc}$ & $22.01 \mathrm{a}$ & $21.79 \mathrm{c}$ & $22.64 \mathrm{~b}$ & $23.5 \mathrm{a}($ & $23.33 \mathrm{a}$ \\
PL $(\mathrm{cm})$ & $( \pm 0.31)$ & $( \pm 0.69)$ & $( \pm 0.63)$ & $( \pm 0.35)$ & $( \pm 0.38)$ & $( \pm 0.43)$ & $\pm 0.34)$ & $( \pm 0.17)$ \\
& $1.22 \mathrm{bc}$ & $1.43 \mathrm{~b}$ & $1.16 \mathrm{c}$ & $1.81 \mathrm{a}$ & $0.46 \mathrm{bc}$ & $0.56 \mathrm{bc}$ & $1.04 \mathrm{a}$ & $0.63 \mathrm{~b}$ \\
WPP $(\mathrm{g})$ & $( \pm 0.14)$ & $( \pm 0.16)$ & $( \pm 0.21)$ & $( \pm 0.10)$ & $( \pm 0.10)$ & $( \pm 0.02)$ & $( \pm 0.07)$ & $( \pm 0.14)$ \\
& $8.45 \mathrm{a}$ & $8.63 \mathrm{a}$ & $7.34 \mathrm{~b}$ & $8.77 \mathrm{a}$ & $7.25 \mathrm{~d}$ & $9.41 \mathrm{~b}$ & $8.38 \mathrm{c}$ & $10.29 \mathrm{a}$ \\
DWS $\left(\mathrm{Mg} \mathrm{ha}^{-1}\right)$ & $( \pm 0.32)$ & $( \pm 0.20)$ & $( \pm 0.09)$ & $( \pm 0.60)$ & $( \pm 0.23)$ & $( \pm 0.37)$ & $( \pm 0.44)$ & $( \pm 0.06)$ \\
& $0.39 \mathrm{c}$ & $0.46 \mathrm{~b}$ & $0.50 \mathrm{a}$ & $0.47 \mathrm{ab}$ & $0.30 \mathrm{~b}$ & $0.27 \mathrm{~d}$ & $0.36 \mathrm{a}$ & $0.29 \mathrm{c}$ \\
HI & $( \pm 0.017)$ & $( \pm 0.005)$ & $( \pm 0.014)$ & $( \pm 0.019)$ & $( \pm 0.008)$ & $( \pm 0.010)$ & $( \pm 0.012)$ & $( \pm 0.008)$ \\
\hline
\end{tabular}

* Means with different letters indicates significantly difference between treatment at 0.05 level by LSD test and \pm value indicate mean standard deviation; GY=grain yield; $\mathrm{PT}=$ productive tiller; $\mathrm{NP}=$ no. of panicle; $\mathrm{FG}=$ filled grain; $\mathrm{TG}=1000$-grains; $\mathrm{PL}=$ panicle length; $\mathrm{WPP}=$ weight per panicle; DWS=dry matter weight straw; $\mathrm{HI}=$ Harvest index. 
The results showed that GY increased significantly $(\mathrm{P} \leq 0.05)$ by $44 \%$ with $\mathrm{RHB}$ amendment in the first cycle, while $46 \%$ in the second crop cycle. Similarly, the PT was greater under RHB application with peak at 10 $\mathrm{Mg} \mathrm{ha}^{-1}(86.25 \%)$ than observed in the control for the first crop cycle. However, in the second crop cycle PT was only significantly affected at 10 and $20 \mathrm{Mg} \mathrm{ha}^{-1}$ of RHB application with peak value of $91.45 \%$. In contrast, NP for the first crop cycle was only statistically significant under application of $5 \mathrm{Mg} \mathrm{ha}^{-1}$ RHB. Meanwhile, NP on RHB amended soil for the second crop cycle was significantly $(\mathrm{P} \leq 0.05)$ higher in all RHB applications compared to the control. Furthermore, NP was not significantly different between plots with or without RHB in the first crop cycle. Unlike in the second crop cycle, NP was significantly increased with the increasing rate of RHB application compared to control. No significant difference was found between treatments for FG in the first crop cycle. However, in the second crop cycle RHB found to have significant effect on FG at application rate of 5 and $10 \mathrm{Mg} \mathrm{ha}^{-1}$ compared with the control. Thousand grain weight significantly increased only in RHB rate of 20 $\mathrm{Mg} \mathrm{ha}^{-1}$ for first crop cycle, while statistically significant at 5 and $10 \mathrm{Mg} \mathrm{ha}^{-1}$ RHB application for second crop cycle. Compared to no RHB amendment, PL was significantly increased by $8 \%$ in the first crop cycle and $7 \%$ in the second crop cycle. Weight per panicle significantly increased when $20 \mathrm{Mg} \mathrm{ha}^{-1} \mathrm{RHB}$ was applied in the first crop cycle. In contrast, for the second crop cycle WPP significantly influences at application rate of $10 \mathrm{Mg} \mathrm{ha}^{-1}$ compared to control. In the first crop cycle, there was no significant difference for the DWS and only significantly increased with application of $20 \mathrm{Mg} \mathrm{ha}^{-1} \mathrm{RHB}$ with an increment of $42 \%$. Rice husk biochar application increased $\mathrm{HI}$ in the first crop cycle at all application rates, while HI significantly responded to RHB when $10 \mathrm{Mg} \mathrm{ha}^{-1}$ were applied.

\section{DISCUSSION}

The RHB application rate used in this study was chosen to study the response on high organic topsoil and did not represent an optimal rate for rice growth. Studies have shown that application of biochar can enhance soil carbon pool, resulting in beneficial effects on soil active organic carbon components, including increase in crop biomass and input of fresh organic carbon, improvement in soil structure, promotion of soil aggregate formation, and provision of an ideal habitat for soil microorganisms (Piccolo et al., 1996; Glaser et al., 2000; Whalley et al, 2006; Steinbeiss, et al, 2009; Yang et al., 2020). Biochar is suggested to improve soil quality, and there are reports of stimulated microbial response and loss of native SOC (Steinbeiss et al., 2009). As reported by Lehmann and Rondon (2006), good agronomic effects in other crops have been achieved with rates of biochar from wood between 0.4 and $8.0 \mathrm{t} \mathrm{C} \mathrm{ha}^{-1}$, but no negative effects have been reported below that of $20 \mathrm{t} \mathrm{C}$ $\mathrm{ha}^{-1}$. The applications of RHB in this study (Table 2) indicated positive effects on soil chemical properties. Results indicated that the RHB amendment showed increment in soil $\mathrm{pH}$ at harvest in high organic rice topsoil for the first crop cycle, but did not exhibit a positive effect in the second crop cycle. The high pH of RHB (8.5) in the first crop cycle could be attributed to an increase in soil $\mathrm{pH}$ in the first crop cycle. Apart from that, the high ash content of RHB (89\%) and liming potential of the biochar used could be 
the reason in increment of soil $\mathrm{pH}$ in the first crop cycle. This result is in accordance with studies done by Masulili et al. (2010), who reported an increase in soil $\mathrm{pH}$ under application of RHB with $\mathrm{pH}$ of 8.5.

The application of RHB showed higher soil TC compared to unamended plot at harvest in the second crop cycle ranging from 136.2 to $157.6 \mathrm{~g} \mathrm{~kg}^{-1}$. This can be explained by the relatively higher $\mathrm{C}$ content (413.23 $\mathrm{g} \mathrm{kg}^{-1}$ ) of the used RHB in the second crop cycle and the cumulative effect of the applied RHB from the first crop cycle. A similar result has been reported by Masulili et al. (2010), who reported high levels of SOM under RHB treated plot although low $\mathrm{C}$ organic content compared to raw rice straw or rice husk. However, lower level of soil TC was observed in the second crop cycle compared with the first crop cycle could be attributed by $\mathrm{C}$ losses through soil decomposition and mineralization which directly link with reducing water table during drought period in the second crop cycle (Figure 1).

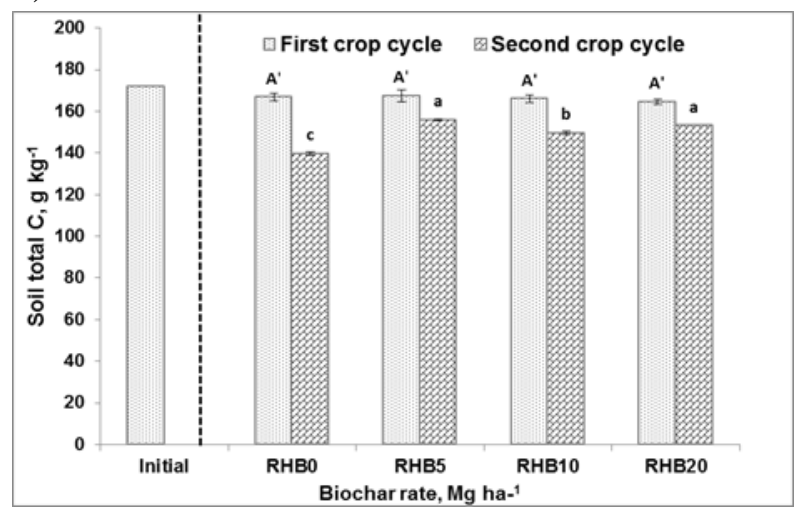

Figure 1: Soil total $\mathrm{C}$ loss in first and second crop cycle (110 DAS) as compared to initial (before treatment), as affected by RHB application and \pm value indicate mean standard deviation.
Studies have shown that the status of soil water content is the key driving factor in the carbon cycle process. Soil water content manifested a significant correlation with the transformation of organic carbon within a certain range of changes. The changes in soil moisture content and its distribution, for example during a period of drought, will have a significant impact on SOC and its active components. This present study was in accordance to the recent findings of Yang et al. (2020), which was also reported previously that soil moisture losses promoted SOC decomposition (Davidson et al, 1998; Flanagan and Johnson, 2005). Amongst all treatments, the least $\mathrm{C}$ loss was recorded at RHB $5 \mathrm{Mg} \mathrm{ha}^{-1}(9 \%)$ and was the highest in control treatment $(19 \%)$ as in Table 5.

RHB addition significantly reduced soil C losses from 9 to $12 \%$ in biochar treated plot in the second cycle. In particular, the increase in soil total $\mathrm{N}$ in this study with RHB application is in agreement with a study by Chan et al. (2008), who reported an increase in soil total $\mathrm{N}$ after application on biochar. Similarly, Dong et al. (2015) reported that soil total $\mathrm{N}$ increases with addition of biochar application by $14.9 \%$ and $11.7 \%$ with and without urea, respectively. Application of RHB amendment exerted a significant effect on enhancing soil available $\mathrm{P}$ by $25 \%$ in the first crop cycle and by $12 \%$ in the subsequent crop cycle. A similar finding by Masulili et al. (2010), who reported that soil available $\mathrm{P}$ was recorded to be higher in RHB treated plots which could be due to high soil $\mathrm{pH}$ under RHB treatment. A similar increase in soil available $\mathrm{P}$ also was reported in previous studies (Lehmann et al., 2002; Yamato et al., 2006). 
Table 5. Soil total C availability after harvest at first and second crop cycle as influenced by RHB application rates.

\begin{tabular}{l|ccc|cc}
\hline \multirow{2}{*}{$\begin{array}{c}\text { RHB } \\
\text { treatment } \\
\left(\mathrm{Mg} \mathrm{ha}^{-1}\right)\end{array}$} & Initial & First crop cycle & Second crop cycle & First crop cycle & $\begin{array}{l}\text { Second } \\
\text { cycle }\end{array}$ \\
\cline { 2 - 6 } crop \\
\hline 0 & 172 & $167( \pm 1.88)$ & $140( \pm 1.33)$ & 2.9 & 18.6 \\
5 & - & $167( \pm 2.81)$ & $156( \pm 0.65)$ & 2.9 & 9.3 \\
10 & - & $166( \pm 1.99)$ & $150( \pm 0.36)$ & 3.5 & 12.8 \\
20 & - & $165( \pm 1.31)$ & $153( \pm 0.77)$ & 4.1 & 11.1 \\
\hline
\end{tabular}

\pm value indicate standard error.

Biochar amendment has previously been shown to increase soil exchangeable cations such $\mathrm{K}, \mathrm{Ca}$ and $\mathrm{Mg}$. In this study, addition of RHB resulted in a $71 \%$ and $4 \%$ increased soil exchangeable $\mathrm{K}$ in the first and second crop cycles, respectively, and this can be associated with the high $\mathrm{K}$ content in the RHB applied (Table 4). Likewise, an increase in soil exchangeable $\mathrm{Mg}$ seems to respond up to 10 $\mathrm{Mg} \mathrm{ha}^{-1} \mathrm{RHB}$ in the first crop cycle, whereas in the second crop cycle only response at 10 and $20 \mathrm{Mg} \mathrm{ha}^{-1} \mathrm{RHB}$ application rate. In addition, positive effect of biochar application on soil exchangeable $\mathrm{Ca}$ was only observed in the second crop cycle at all RHB application rates. A similar finding has also been reported in previous studies by Belyaeva et al. (2012), who reported an increment in soil exchangeable $\mathrm{K}$, $\mathrm{Mg}$ and $\mathrm{Ca}$ in pot study using poultry manure biochar.

In both crop cycles, RHB application had a positive effect on plant $\mathrm{N}$ uptake which in accordance with Huang et al. (2014), who reported an increased fertilizer $\mathrm{N}$ uptake by rice by approximately $23-27 \%$. This might contribute to the increased availability of soil $\mathrm{N}$ from decomposition of indigenous organic matter (apart from the fertilizer $\mathrm{N}$ ) for $\mathrm{N}$ uptake with application of biochar. Similarly, $\mathrm{P}$ and $\mathrm{K}$ uptake in both crop cycles were slightly higher in biochar application with an increment of $18 \%$ and $31 \%$, respectively as compared to control treatment. This might be due to great availability of nutrients content in the soil, which is in accordance with studies done by Major et al. (2010). In addition, an increase in plant K uptake also was reported with biochar application in cowpea crop in a pot study by Lehmann et al. (2003). However, an unusual long drought event that occurred during the second crop cycle led to a significantly lower rice plant uptake of $\mathrm{P}$ and $\mathrm{K}$, which might be due to the abiotic stress conditions.

In comparison to study by Zhang et al. (2012), rice yield increased $10 \%$ in first crop cycle and by 9.5 to $29 \%$ in subsequent cycle using wheat straw biochar on clay soil, whereas in this study, grain yield increased significantly by $63 \%$ and $51 \%$ with a threshold value of $16 \mathrm{Mg} \mathrm{ha}^{-1} \mathrm{RHB}$ (8.02 $\mathrm{Mg} \mathrm{ha}^{-1}$ ) and $14 \mathrm{Mg} \mathrm{ha}^{-1}$ of RHB (4.55 Mg $\mathrm{ha}^{-1}$ ) in first and second crop cycle, respectively (Figure 4). Although grain yield in the second crop cycle seemed to decrease as compared to the first crop cycle, which might be a consequence of an unusual long drought period during this growing season. 
Table 6. Correlation between soil properties, nutrient uptake and rice yield parameters to grain yield of two crop cycles.

\begin{tabular}{|c|c|c|}
\hline Variables & $\begin{array}{c}\text { First crop cycle (Grain yield) } \\
\mathrm{Mg} \mathrm{ha}^{-1}\end{array}$ & $\begin{array}{c}\text { Second crop cycle (Grain yield) } \\
\mathrm{Mg} \mathrm{ha}^{-1}\end{array}$ \\
\hline Soil pH & $0.64 * *$ & ns \\
\hline Available $\mathrm{P}\left(\mathrm{mg} \mathrm{kg}^{-1}\right)$ & ns & $0.63 * *$ \\
\hline Exchangeable $\mathrm{K}\left(\mathrm{mg} \mathrm{kg}^{-1}\right)$ & $0.72 * * *$ & $0.57 *$ \\
\hline Exchangeable $\mathrm{Mg}\left(\mathrm{mg} \mathrm{kg}^{-1}\right)$ & $\mathrm{ns}$ & $0.85^{* * *}$ \\
\hline Exchangeable $\mathrm{Ca}\left(\mathrm{mg} \mathrm{kg}^{-1}\right)$ & $\mathrm{ns}$ & $0.61 *$ \\
\hline P uptake $\left(\mathrm{kg} \mathrm{ha}^{-1}\right)$ & $\mathrm{ns}$ & $0.72 * *$ \\
\hline $\mathrm{K}$ uptake $\left(\mathrm{kg} \mathrm{ha}^{-1}\right)$ & $0.82 * * *$ & $0.75^{* * *}$ \\
\hline $\mathrm{Mg}$ uptake $\left(\mathrm{kg} \mathrm{ha}^{-1}\right)$ & $0.51 *$ & $0.51^{*}$ \\
\hline Cu uptake $\left(\mathrm{kg} \mathrm{ha}^{-1}\right)$ & $0.70 * *$ & ns \\
\hline Zn uptake $\left(\mathrm{kg} \mathrm{ha}^{-1}\right)$ & ns & $0.52 *$ \\
\hline Productive tiller (\%) & $0.69 * *$ & $0.56^{*}$ \\
\hline No. of panicle & ns & $0.94 * * *$ \\
\hline Panicle length $(\mathrm{cm})$ & $0.72 * *$ & $0.86^{* * *}$ \\
\hline Weight per panicle $(\mathrm{g})$ & ns & $0.84 * * *$ \\
\hline
\end{tabular}

Nevertheless, grain yield under RHB The improvement in soil chemical properties, treatments were observed to be higher as viz. soil $\mathrm{pH}$, available $\mathrm{P}$, exchangeable $\mathrm{K}$ and compared to control treatment despite growing under stressconditions. This can be attributed to the increase in soil water retention with biochar application (Karhu et al., 2011) which is incredibly important during drought. The optimum soil $\mathrm{pH}$ for good rice growth was reported within range of 5.5 to 7.0 (Pedro et al., 1990). A similar increase in grain yield were also reported in previous studies, such in upland rice using wood biochar in Northern Laos (Asai et al., 2009) and on acid sulfate soil using RHB in West Kalimantan, Indonesia (Masulili et al., 2010) and on gley paddy soil using bamboo biochar and RHB in Zhejiang, China (Zhang et al., 2010). The increase in grain yield for both crop cycles as compared to control treatment seemed to be positively correlated with a slight increase in percent of PT, PL and WPP (Table 6). exchangeable $\mathrm{Mg}$, and plant macronutrients $(\mathrm{N}, \mathrm{P}$ and $\mathrm{K}$ ) uptake by rice plants seems to be a consequence of an increase in grain yield.

Additionally, PT showed an increase of 7 to $8 \%$ in RHB amended plots for both crop cycles in comparison to control plots Such sustainable increasing effect could be supported by other field studies, such as Masulili et al., (2010), who reported increase in PT under RHB treatment on acid sulfate soil in West Kalimantan, Indonesia. The higher PT in biochar amended plots compared to unamended plots may contribute to an increase in plant nutrient uptake, especially of $\mathrm{N}$, and the availability of nutrients in soil. Therefore, RHB application exerted significant positive effects on NP, FG, PL and WPP in both crop cycles. The positive effect of RHB in NP was in accord with studies by Singla et al., (2014), who reported an increase in NP using biochar combined with ammonium sulfate using

Journal of Wetlands Environmental Management 
pot study in Chiba, Japan. In comparison to the first crop cycle, lower NP, PL and WPP produced in the second crop cycle were clearly affected by the long drought period during this growing season. The DMWs in the first crop cycle had no positive effect toward RHB application, while in the second crop cycle DMWs found to increase under all RHB application rates. Other than the increase in yield parameters by RHB addition, the difference in the HI for first crop cycle was significant compared to control; however, the in second cropping season the HI showed response up to $5 \mathrm{Mg} \mathrm{ha}^{-1}$ as compared to control treatment, which is contrary to the studied by Tammeorg et al. (2014) on faba bean.

\section{CONCLUSION}

The present study showed high grain yield production with RHB application in the first and second crop cycles, with an increment of $44 \%$ and $46 \%$, respectively. RHB application correspondingly increased yield variables including productive tillers, panicle length and weight per panicle. Application of RHB significantly improved N, P, and K uptake. Further studies are proposed, especially to determine the long term effects of RHB soil amendment on rice cultivated in soils with high organic matter.

\section{REFERENCES}

Asai, H., Samson, B.K., Stephan, H.M., Songyikhangsuthor, K., Homma, K., Kiyono, Y., Inoue, Y., Shiraiwa, T., \& Horie, T. (2009). Biochar amendment techniques for upland rice production in Northern Laos 1. Soil physical properties, leaf SPAD and grain yield. Field Crops Research, 111, 81-84.

Belyaeva, O. N., \& Haynes, R. J. (2012). Comparison of the effects of conventional organic amendments and biochar on the chemical, physical and microbial properties of coal fly ash as a plant growth medium. Environmental Earth Sciences, 66(7), 19871997.

Bernas, 2020. Accessed on 11th August 2020. http://www.bernas.com.my/bernas/index.ph p/ricepedia/rice-process

Chan KY, Van Zwieten L, Meszaros I, Downie A, Joseph S (2008) Using poultry litter biochars as soil amendments. Aust J Soil Res 46:437-444.

de Melo Carvalho, M. T., Madari, B. E., Bastiaans, L., van Oort, P. A. J., Heinemann, A. B., da Silva, M. A. S., . \& Meinke, H. (2014). Biochar improves fertility of a clay soil in the Brazilian Savannah: short term effects and impact on rice yield. Journal of Agriculture and Rural Development in the Tropics and Subtropics (JARTS), 114(2), 101-107.

Davidson, E.; Belk, E.; Boone, R. Soil water content and temperature as independent or confounded factors controlling soil respiration in a temperate mixed hardwood forest. Glob. Chang. Biol. 1998, 4, 217-227. 
Deenik, J., Diarra, A., Uehara, G., Campbell,

S., Sumiyoshi, Y., Antal Jr., M., 2011.

Charcoal ash and volatile matter effects on

soil properties and plant growth in an acid

Ultisol. Soil Science 176, 336-345.

Department of Statistics Malaysia, 2016.

Population distribution and basic

demographic. Accessed on 11th August 2020.

https://www.dosm.gov.my/v1/index.php?r =column/cthemeByCat\&cat=164\&bul_id= Tm5OaVh6RFpFM2VGOTIrZzltbWg3Q T09\&menu_id=Z0VTZGU1UHBUT1VJ

MFlpaXRRR0xpdz09

Department of Agriculture, Peninsular Malaysia. 2020. Paddy Statistics of Malaysia, 2015. Putrajaya: Department of Agriculture, Peninsular Malaysia.

Flanagan, L.; Johnson, B. Interacting effects of temperature, soil moisture and plant biomass production on ecosystem respiration in a northern temperate grassland. Agric. For. Meteorol. 2005, 130, 237-253.

Glaser, B.; Balashov, E.; Haumaier, L.; Guggenberger, G.; Zech, W. Black carbon in density fractions of anthropogenic soils of the Brazilian Amazon region. Org. Geochem. 2000, 31, 669-678.

Haefele, S. M., Konboon, Y., Wongboon, W., Amarante, S., Maarifat, A. A., Pfeiffer, E. M. \& Knoblauch, C. (2011). Effects and fate of biochar from rice residues in ricebased systems. Field Crops Research, 121, 430-440.

Huang, M., Yang, L., Qin, H., Jiang, L., \& Zou, Y. (2014). Fertilizer nitrogen uptake by rice increased by biochar application. Biology and fertility of soils, 50(6), 997-
1000.

Index Mundi, 2020, Agriculture. Accessed on 11th August 2020. https://www.indexmundi.com/agriculture/?c ommodity=milled-rice $\&$ graph $=$ production

Karhu, K., Mattila, T., Bergström, I., Regina, K., 2011. Biochar addition to agricultural soil increased $\mathrm{CH} 4$ uptake and water holding capacity results from a short-term pilot field study. Agriculture, Ecosystems and Environment 140, 309-313.

Lehmann, J., da Silva Jr., J.P., Rondon, M., Steiner, C., Nehls, T., Zech, W., Glaser, B., 2002. Nutrient availability and leaching in an archaeological Anthorosol and a Ferralsol of the Central Amazon basin: fertilizer, manure and charcoal amendments. Plant and Soil 249, 343-357.

Lehmann J, Silva JJP, Steiner C, Nehls T, Zech W, Glaser B (2003) Nutrient availability and leaching in an archaeological Anthrosol and a Ferralsol of the Central Amazon basin: fertilizer, manure and charcoal amendments. Plant Soil 249:343-357.

Lehmann J, Gaunt J, Rondon M (2006) Biochar sequestration in terrestrial ecosystems - a review. Mitig Adapt Strategies Glob Chang 11:395-419.

Lehmann, J., 2007. A handful of carbon. Nature 447, 143-144. Laird, D.A., 2008. The charcoal vision: a win-win-win scenario for simultaneously producing bioenergy, permanently sequestering carbon, while improving soil and water quality. Agron. J. 100,178-181.

Lehmann, J., Joseph, S., 2009. Biochar for environmental management: an introduction. In: Lehmann, J., Joseph, S. (Eds.), Biochar for Environmental 
Management: Science and Technology. Earthscan, London, pp. 1-12.

Major, J., Rondon, M., Molina, D., Riha, S.J., Lehmann, J., 2010. Maize yield and nutrition during 4 years after biochar application to a Colombian savanna oxisol. Plant and Soil 333, 117-128.

Masulili, A., Utomo, W. H. \& Syekhfani. (2010). Rice husk biochar for rice based cropping system in acid soil 1 . The characteristics of rice husk biochar and its Influence on the properties of acid sulfate soils and rice growth in West Kalimantan, Indonesia. Journal of Agriculture Science (Canada), 3, 25-33.

Pedro D. Sangatanan and Rone Sangatanan, Soil management (1990) ISBN 971-23 0581-3 books.google.com.ph/books?i

Piccolo, A.; Pietramellara, G.; Mbagwu, J. Effects of coal derived humic substances on water retention and structural stability of Mediterranean soils. Soil Use Manag. 1996, 12, 209-213.

Rondon, M.A., Lehmann, J., Ramirez, J., Hurtado, M., 2007. Biological nitrogen fixation by common beans (Phaseolus vulgaris L.) increases with biochar additions. Biol. Fertil. Soils 43, 699-708.

Woolf D, Amonette JE, Stree-Perrott FA, Lehmann J, Joseph S (2010) Sustainable biochar to mitigate global climate change. Nat Comm 1:56.

Singla A, Iwasa H, Inubushi K (2014) Effect of biogas digested slurry based-biochar and digested liquid on $\mathrm{N} 2 \mathrm{O}, \mathrm{CO} 2$ flux and crop yield for three continuous cropping cycles of komatsuna (Brassica rapa var. perviridis). Biol. Fertil. Soils 50:12011209.
Steinbeiss, S.; Gleixner, G.; Antonietti, M. Effect of biochar amendment on soil carbon balance and soil microbial activity. Soil Biol. Biochem. 2009, 41, 1301-1310.

Steiner, C., Teixeira, W.G., Lehmann, J., Nehls, T., de Macedo, J.L.V., Blum, W.E.H., Zech, W., 2007. Long term effects of manure, charcoal and mineral fertilization on crop production and fertility on a highly weathered Central Amazonian upland soil. Plant and Soil 291, 275-290.

Tammeorg, P., Simojoki, A., Mäkelä, P., Stoddard, F. L., Alakukku, L., \& Helenius, J. (2014). Biochar application to a fertile sandy clay loam in boreal conditions: effects on soil properties and yield formation of wheat, turnip rape and faba bean. Plant and soil, 374(1-2), 89-107.

Van Zwieten, L., Kimber, S., Morris, S., Chan, K.Y., Downie, A., Rust, J., Joseph, S., Cowie, A., 2010. Effects of biochar from slow pyrolysis of paper mill waste on agronomic performance and soil fertility. Plant and Soil 327, 235-246.

Yamato, M., Okimori, Y., Wibowo, I. F., Anshori, S., \& Ogawa, M., 2006. Effects of the application of charred bark of Acacia mangium on the yield of maize, cowpea and peanut, and soil chemical properties in South Sumatra, Indonesia. Soil Science and Plant Nutrition, 52(4), 489-495.

Yang, S., Chen, X., Jiang, Z, Ding, J., Sun, X., $\mathrm{Xu}$, J., 2020. Effects of biochar application on soil organic carbon composition and enzyme activity in paddy soil under watersaving irrigation. International Journal of Environmental Research and Public Health. 17, 333; doi:10.3390/ijerph17010333 www.mdpi.com/journal/ijerph 
Zhang, A., Cui, L., Pan, G., Li, L., Hussain, Q., Zhang, X., Zheng, J., Crowley, D., 2010. Effect of biochar amendment on yield and methane and nitrous oxide emissions from a rice paddy from Tai Lake plain, China. Agriculture. Ecosystems and Environment 139, 469475.

Zhang, A.F., Bian, R.J., Pan, G.X., Cui, L.Q., Hussain, Q., Li, L.Q., Zheng, J.W., Zheng, J.F., Zhang, X.F., Han, X.J., Yu, X.Y., 2012. Effects of biochar amendment on soil quality, crop yield and greenhouse gas emission in a Chinese rice paddy: a field study of 2 consecutive rice growing cycles. Field Crops Res. 127, 153-160. 